\title{
DOSEAMENTO DO FOSFORO EM MEDICAMENTOS REIDRATANTES
}

Alnei Ramos Prochnow, Francisco Xavier Pillar e Glaucia Ustra Soares Departamento de Física. Centro de Ciências Naturais e Exatas. UFSM. Santa Maria; RS.

João Fernandes Magalhães e Maria Inês Rocha Miritello Santoro Departamento de Farmäcia. Faculdade de Ciēncias Farmacēuticas. USP. São Paulo. SP.

\section{RESUMO}

0 teor do fósforo em soluções reidratantes foi determinado pelo método do azul de molibdênio, usando o sulfato de hidrazina co mo agente redutor. Além dos parāmetros espectrofotométricos foi veri ficada a interferência de ions que, em geral, estão presentes neste tipo de formulação famacêutica.

\section{SUMMARY}

PROCHNOW,A.R.; PILLAR,F.X.; SOARES,G.U.; MAGALHAES, J.F. and SANTORO, M.I.R.M., 1980. Determination of phosphorus in reidratant medicaments. Ciēncia e Natura (2): 19-27.

The phosphor content in rehydrating solutions it was deter mined through of the molybdenium blue method, using hydrazine sulfate as a reducing agent. Besides the spectrophotometric parameters itwas studied the ions interference that generally are present in this kind of pharmacentical formulation.

\section{INTRODUÇAO}

A desidratação freqüente ē um dos problemas sanitārios,par ticularmente, nas regiões de clima tropical. Entre as medidas terapéu ticas para o tratamento deste quadro clinico destacam-se os reidratan tes constituídos de soluções, contendo diversos îons. Normalmente,as soluções são fornecidas prontas ou são preparadas no momento do uso, isso a partir de uma solução concentrada, como também de uma amostra da mistura de sais na forma de pō.

Nesses medicamentos, devido à natureza dos compostos, é co mum ocorrer incompatibilidades quimicas que ocasionam problemas de or dem farmacotēcnica ou de estabilidade.

Devemos considerar, tambēm, que os problemas são acrescidos de caréncia de métodos analíticos, suficientemente seletivos e aces sîveis. A reatividade química dos diversos ỉons provoca interferēn cia múltipla no andamento dos processos quantitativos. Assim, é fre qüente o impedimento das operações analîticas ou nỉtida influéncia 
nos resultados.

Em vista disso, passamos a estudar os problemas inerentes ao controle de qualidade destes produtos, iniciando pela determinação do fósforo que, em razão de sua capacidade de complexação, atua como um dos principais obstáculos.

\section{REVISAOO DA LITERATURA}

Os medicamentos reidratantes são formulados com o objetivo de restituir ao organismo a àgua e alguns íons inorgânicos, que são eliminados no processo de desidratação.

os principais métodos para determinação do fósforobaseiamse em reações do ỉon ortofosfato. Para amostras, contendo grande pro porção do elemento, são empregados métodos gravimētricos e volumētri cos com molibdato de amōnio $(8,12)$.

As amostras com pequenas quantidades de fōsforo são anal 1 sadas por mētodos colorimētricos e espectrofotométricos. Os tratamen tos analiticos variam muito em função da natureza das amostras edos objetivos visados. Quase sempre são necessārias adaptações para re solver casos especĩficos. DONALD et alii (6) empregaram a reação do ĩon ortofosfato com o àcido molíbdico, contendo vanádio. o produto da reação tem coloração amarela, sendo a leitura espectrofotométrica das soluções realizadas entre 420 a $480 \mathrm{~nm}$.

A determinação do arsênico pelo àcido molíbdico e posterior redução com sulfato de hidrazina seguiu o método indicado por MORRIS e CALVERY (11).

Posteriormente, a mesma reação foi sugerida por OHLWEILLER (12) para anālise fotométrica do fósforo. A redução seletiva do āci do fosfomolíbdico, formado na reação, resulta em um produto de compo sição indefinida de intensa coloração azul, proporcional à concentra ção de fósforo.

Entretanto, uma das limitações do método é a interferência provocada por diversos ĩons orgānicos e inorgānicos.

Surgiram muitas variações desse método, mas na maioria das vezes referem-se à substituição do agente redutor. 0 cloreto estanoso foi usado por SCHAFFER e colaboradores (13), DICKMAN e BRAY (5)e por SHAW (15). O àcido 1-amino-2-naftol-4sulfōnico foi empregado por BAR TLETT (2) e por KIRK,PATRICK \& BISHOP (9). 0 sulfato de p-metilami nofenol foi escolhido por DREWES (7) e por SEE \& FITT (14) e a o-fe nilenodiamina foi indicada por MORIN \& PROX (10).

A determinação espectrofotométrica no ultravioleta do āc

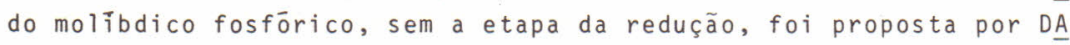
LY \& ERTINGSHAUSEN (4) e por AMADOR \& URBAN (1). 
Nos medicamentos reidratantes, normalmente, estão presen tes alguns ions que interferem no método do azul de molibdēnio, có mo o cloreto, acetato, citrato, cālcio, magnésio e outros. Mesmo as sim, como as características do método são muito favoráveis para apli cação rotineira, procuramos estudar quais as possibilidades de seu aproveitamento na anālise do fósforo.

0 trabalho foi desenvolvido com o objetivo de verificarmos a relação entre concentração e absorbāncia, a interferēncia dos ỉons presentes numa preparação farmacēutica lĩquida e a aplicação do mé todo nessa preparação.

\section{MATERIAL E METODOS}

Reagentes

Os reagentes empregados foram: Hidrogenofosfato de potās sio (Esta solução contēm $0,05 \mathrm{mg}$ de fósforo por ml). Solução de mo libdato de sódio: $2,5 \%$ em ācido sulfúrico $10 \mathrm{~N}$. Solução de citrato de sōdio: $5 \mathrm{mg}$ por ml. Solução de sulfato de hidrazina: $0,15 \%$ em ägua deionizada.

Preparação Farmacêutica

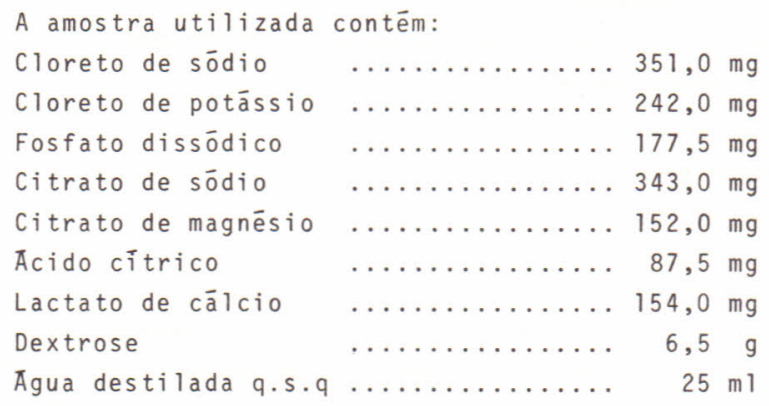

Método

Foi estudado o método colorimétrico utilizando um espectrofotômetro para o visivel e ultravioleta. Observamos as indicações de OHLWEILLER (12) com algumas modificações na concentração de leitura, usando āgua destilada como solvente. O espectro foi obtido a partir de uma solu ção padrão de fosfato a $1 \mu \mathrm{g} / \mathrm{ml}$.

Reta de Calibração

Inicialmente, determinou-se a curva de Ringbom, partindo de 20 (vinte) soluções cujas concentrações variavam de 0,4 a $4,2 \mu \mathrm{g}$ $/ m l$ de fósforo; foram utilizados 20 (vinte) balões volumétricos de $50 \mathrm{ml}$ de capacidade, para os quais foram transferidas quantidades cres centes de uma solução mãe de fosfato a $4 \mu \mathrm{g} / \mathrm{ml}$ de fósforo. A seguir foram adicionados aos balões $5 \mathrm{ml}$ da solução de molibdato de sódio, 
2 ml da solução de sulfato de hidrazina, completando-se o volume com àgưa deionizada.

As leituras foram efetuadas a $830 \mathrm{~nm}$, usando-se como bran co uma solução de sulfato de hidrazina, molibdato de sōdio e água deionizada. Com os resultados obtidos foi traçada a curva de Ringbom (absorvēncia versus log da concentração). Partindo dos dados obtidos, selecionaram-se 10 (dez) concentrações com as respectivas obsorbān ciaś e foi calculada a reta de calibração pelo método dos mỉnimos quadrados, com as considerações estatísticas necessārias.

Aplicą̧ão do método na Análise do Medicamento

$\mathrm{Da}$ amostra do medicamento foram retiradas aliquotas de $10 \mathrm{ml}$ com $2 \mu \mathrm{g} / \mathrm{ml}$ de fósforo (concentração ideal da curva de Ringbom) e transferida para balões de $50 \mathrm{ml}$. Foram adicionados a cada balão $5 \mathrm{ml}$ de molibdato de sódio, $2 \mathrm{ml}$ de sulfato de hidrazina,completando-seo volume com água deionizada. A seguir, as misturas foram aquecidas em banho-maria durante 10 minutos. Foram realizadas 10 anālises da amos tra do medicamento. As leituras foram efetuadas a $830 \mathrm{~nm}$, usando-se como branco uma solução, contendo sulfato de hidrazina, molibdato de sōdio e àgua deionizada.

0s resultados foram comparados com um padrão tratado da mes ma maneira e analisado estatísticamente.

Influência do ion citrato

Para verificar a influencia do ion citrato, foram feitas 5 (cinco) preparações, tomando-se para cada uma delas uma alíquota de $10 \mathrm{ml}$ da solução padrão de fosfato $2 \mu \mathrm{g} / \mathrm{ml}$, adicionando-se em ca da uma $2 \mathrm{ml}$ de sulfato de hidrazina, $5 \mathrm{ml}$ de molibdato de sódio e, colocando-se $1 \mathrm{ml}, 3 \mathrm{ml}, 4 \mathrm{ml}, 5 \mathrm{ml}$, respectivamente, de citrato de sōdio a $100 \mu \mathrm{g} / \mathrm{ml}$, completando-se o volume para $50 \mathrm{ml}$ de água.

os resultados obtidos foram comparados, espectrofotométri camente, com uma solução que não continha citrato e tratada da mes ma maneira. Experiēnciasidēnticas foram realizadas com os ions clore to e lactato.

RESULTADOS

0 espectro de absorção do fosfato pelo método do azul de molibdēnio apresentou pico de máximo em $830 \mathrm{~nm}$.

Na Tabela I estão reunidos os valores referentes às expe riências para a determinação da Curva de Ringbom, ilustrada na Figu ra 1 .

Na Tabela II, podem ser vistos os valores empregados para - cálculo da reta de calibração; esta pode ser observada na figura 2. A Tabela III, apresenta o resumo da anālise estatỉstica dos 
TABELA I. RESULTADOS DA EXPERIENCIA PARA A DETERMINAÇAO DA CURVA DE RINGBOM.

\begin{tabular}{|c|c|c|c|c|c|c|}
\hline TUBO & $\begin{array}{c}\text { PADRAO } \\
\text { EM } \\
\text { m1 }\end{array}$ & $\begin{array}{c}\text { REAGENTE } \\
\text { EM } \\
\text { m l }\end{array}$ & $1-T$ & A & $T$ & $\begin{array}{c}\text { CONCENTRAÇAO } \\
\text { DE FOSFORO } \\
\mu \mathrm{g} / \mathrm{m} 1\end{array}$ \\
\hline 1 & 1,0 & 7,0 & 11,5 & 0,053 & 88,5 & 0,4 \\
\hline 2 & 1,5 & 7,0 & 18,0 & 0,086 & 82,0 & 0,6 \\
\hline 3 & 2,0 & 7,0 & 23,5 & 0,116 & 76,5 & 0,8 \\
\hline 4 & 2,5 & 7,0 & 32,0 & 0,167 & 68,0 & 1,0 \\
\hline 5 & 3,0 & 7,0 & 34,0 & 0,180 & 66,0 & 1,2 \\
\hline 6 & 3,5 & 7,0 & 41,5 & 0,232 & 58,5 & 1,4 \\
\hline 7 & 4,0 & 7,0 & 45,0 & 0,259 & 55,0 & 1,6 \\
\hline 8 & 4,5 & 7,0 & 47,5 & 0,279 & 52,5 & 1,8 \\
\hline 9 & 5,0 & 7,0 & 52,0 & 0,318 & 48,0 & 2,0 \\
\hline 10 & 5,5 & 7,0 & 55,5 & $0,35]$ & 44,5 & 2,2 \\
\hline 11 & 6,0 & 7,0 & 58,0 & 0,376 & 42,0 & 2,4 \\
\hline 12 & 6,5 & 7,0 & 62,0 & 0,420 & 38,0 & 2,6 \\
\hline 13 & 7,0 & 7,0 & 64,5 & 0,449 & 35,5 & 2,8 \\
\hline 14 & 7,5 & 7,0 & 67,5 & 0,488 & 32,5 & 3,0 \\
\hline 15 & 8,0 & 7,0 & 70,5 & 0,530 & 29,5 & 3,2 \\
\hline 16 & 8,5 & 7,0 & 71,5 & 0,545 & 28,5 & 3,4 \\
\hline 17 & 9,0 & 7,0 & 75,5 & 0,610 & 24,5 & 3,6 \\
\hline 18 & 9,5 & 7,0 & 77,0 & 0,638 & 23,0 & 3,8 \\
\hline 19 & 10,0 & 7,0 & 78,5 & 0,667 & 21,5 & 4,0 \\
\hline 20 & $\ldots$ & 7,0 & 80,5 & 0,709 & 19,5 & $\ldots$ \\
\hline
\end{tabular}

1-T: Absorvêncìa

A : Absorbancia

T: Transmitāncia

valores obtidos para a reta de calibração.

os resultados do doseamento do medicamento constam na Ta bela IV.

A Tabela $V$ apresenta o resumo da anālise estatística dos da dos obtidos no doseamento do medicamento, através do método colorí métrico.

Nas experiências para verificar a interferência dos ions citrato, lactato e cloreto, não foram observadas diferenças signifi cativas nos resultados encontrados.

0 tratamento estatístico para os resultados do doseamento do medicamento estudado foi o seguinte:

cálculo do desvio padrão: $s(\bar{\Delta})=\sqrt{s^{2}(\bar{\Delta})}$ 
TABELA II. VALORES PARA O CALCULO DA RETA DE CALIBRAÇÃO.

\begin{tabular}{ccccc}
\hline \hline $\begin{array}{c}X \\
\text { ABSORBANCIA }\end{array}$ & \multicolumn{1}{c}{$\mathrm{y}_{\mathrm{m} 1}$} & $\mathrm{x}^{2}$ & $y^{2}$ & $\mathrm{xy}$ \\
\hline 0,232 & 1,4 & 0,053824 & 1,96 & 0,3248 \\
0,259 & 1,6 & 0,067081 & 2,56 & 0,4144 \\
0,279 & 1,8 & 0,077841 & 3,24 & 0,5022 \\
0,318 & 2,0 & 0,101124 & 4,00 & 0,6360 \\
0,351 & 2,2 & 0,123201 & 4,48 & 0,7722 \\
0,376 & 2,4 & 0,141376 & 5,76 & 0,9024 \\
0,420 & 2,6 & 0,176400 & 6,76 & 1,0920 \\
0,449 & 2,8 & 0,201601 & 7,84 & 1,2572 \\
0,488 & 3,0 & 0,238144 & 9,00 & 1,4640 \\
0,530 & 3,2 & 0,280900 & 10,24 & 1,6960 \\
\hline \multirow{2}{*}{3,702} & 23,00 & 1,461492 & 56,20 & 9,0612 \\
\hline
\end{tabular}

TABELA III. RESUMO DOS VALORES OBTIDOS PARA A RETA DE CALIBRAÇAO PE LO METODO DOS MINIMOS QUADRADOS.

\begin{tabular}{cccccccc}
\hline METODO & b & a & teste significância & & \multicolumn{2}{c}{ Precisão } \\
& & $t$ & $a$ & $b^{\prime}$ & Absoluta & Relativa \\
\hline Visível & 6,0058278 & 0,0766425 & 0,07431184 & n/signif. & 6,1999655 & 0,0208 & 0,10078 \\
\hline
\end{tabular}

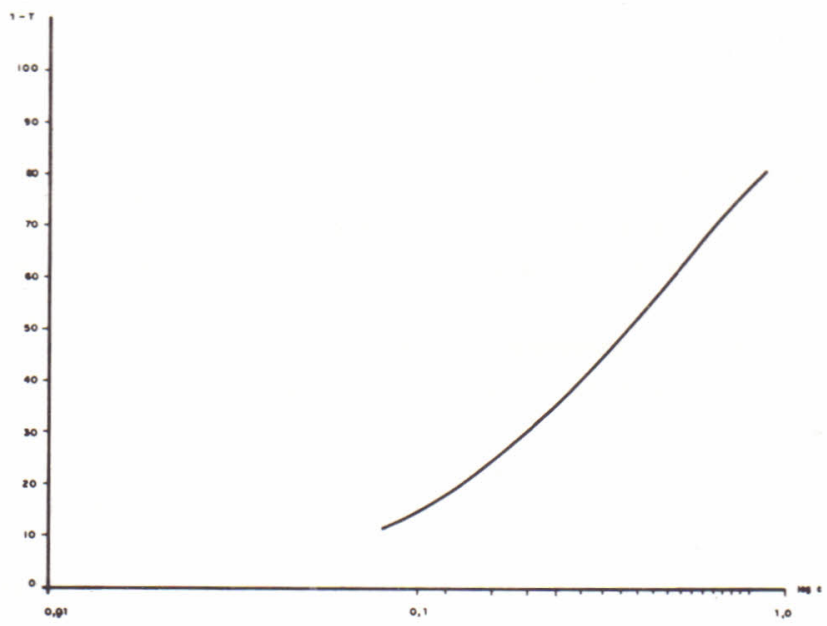

Figura 1. Curva de Ringbom para o fosfato, a $830 \mathrm{~nm}$. 
TABELA IV. RESULtAdOS DO DOSEAMENTO DO MEDICAMENTO CONTENDO 177,5 $\mathrm{mg} / 25 \mathrm{~m} f$ DE FOSFATO DISSODICO.

\begin{tabular}{ccccccc}
\hline \hline \multirow{2}{*}{ AMOSTRA } & $T$ & $\mathrm{~A}$ & \multicolumn{5}{c}{ VALORES } & ENCONTRADOS \\
& & & $\mathrm{x} \mathrm{mg} / 25 \mathrm{ml}$ & $\Delta$ & $\Delta^{2}$ & $\%$ \\
\hline 1 & 23,0 & 0,638 & 182,79340 & 5,2934 & 28,020084 & 102,98219 \\
2 & 23,5 & 0,628 & 180,11855 & 2,61855 & 6,8568041 & 101,47523 \\
3 & 23,0 & 0,638 & 182,79340 & 5,2934 & 28,020084 & 102,98219 \\
4 & 24,5 & 0,610 & 174,93544 & $-2,56456$ & 6,576968 & 98,555177 \\
5 & 24,0 & 0,619 & 177,50000 & 0,00000 & 0,000000 & 100,00000 \\
6 & 24,5 & 0,610 & 174,93544 & $-2,56456$ & 6,576968 & 98,555177 \\
7 & 25,0 & 0,602 & 172,42268 & $-5,07732$ & 25,779178 & 97,139538 \\
8 & 24,5 & 0,610 & 174,93544 & $-2,56456$ & 6,576968 & 98,555177 \\
9 & 24,5 & 0,610 & 174,93544 & $-2,56456$ & 6,576968 & 98,555177 \\
10 & 24,0 & 0,619 & 177,50000 & 0,00000 & 0,000000 & 100,000000 \\
PADRAO & 4,0 & 0,619 & 177,50000 & 0,00000 & 0,000000 & 100,000000 \\
\hline
\end{tabular}

T: Transmitância

A : Absorvāncia

$X$ : Valores das leituras individuais.

$\Delta$ : Diferença entre o valor encontrado pelo doseamento e o valor teó rico ná tomada de ensaio.

TABELA V. RESULTADOS DA ANALLISE ESTATISTICA DOS DADOS OBTIDOS, ATRA VES DO DOSEAMENTO DO FOSFATO DISSODICO, EM MEDICAMENTOS REHIDRATANTES, PELO METODO COLORIMETRICO.

\begin{tabular}{lccrcc}
\hline MEDICAMENTO & TESTE & VICIO ABSOLUTO & VICIO & PRECISAO \\
\hline REHIDRATANTE & $\mathrm{t}$ & MEDIA EXPER. & INTERVALO & RELATIV0 \% & RELATIVA \% \\
& $\begin{array}{c}0,1888187 \\
\text { não signif. }\end{array}$ & -213021 & $\begin{array}{l}0,213021 \pm \\
2,5517106\end{array}$ & 0,120156 & 2,0121576 \\
\hline
\end{tabular}

cālculo de "t" student: $t=\frac{\bar{\Delta}}{s(\bar{\Delta})}$

vicio absoluto: média experimental das diferenças entre os valores esperados e encontrados: $\Delta \frac{\Sigma \Delta}{n}$

intervalo do vício verdadeiro para probabilidade de $95 \%$ : $\bar{\Delta} \pm 2,262 \mathrm{~s}(\bar{\Delta})$

cālculo do vīcio relativo $100 \cdot \frac{\bar{\Delta}}{\bar{x}}$

DISCUSSAOO

Embora o método de azul de molibdênio, para doseamento de fōsforo, tenha sido descrito hā bastante tempo, não encontramos na literatura informações mais detalhadas sobre os principais parâmetros 
espectrofotomētricos.

Os resultados experimentais mostraram excelente relação en tre a absorbância e concentração.

A determinação de Curva Ringbom possibilitou a definição exata do intervalo de concentração no qual o método é vālido.

A aplicação do método no medicamento analisado, não sofreu interferência dos ions cloreto, citrato e lactato. Provavelmente, nes te caso, a concentração dos ions não foi suficiente para provocar al gum tipo de interferência.

Por outro lado, a grande sensibilidade do método não com prometeu a exatidão e a precisão, ao contrārio do que ocorre com ou tros métodos.

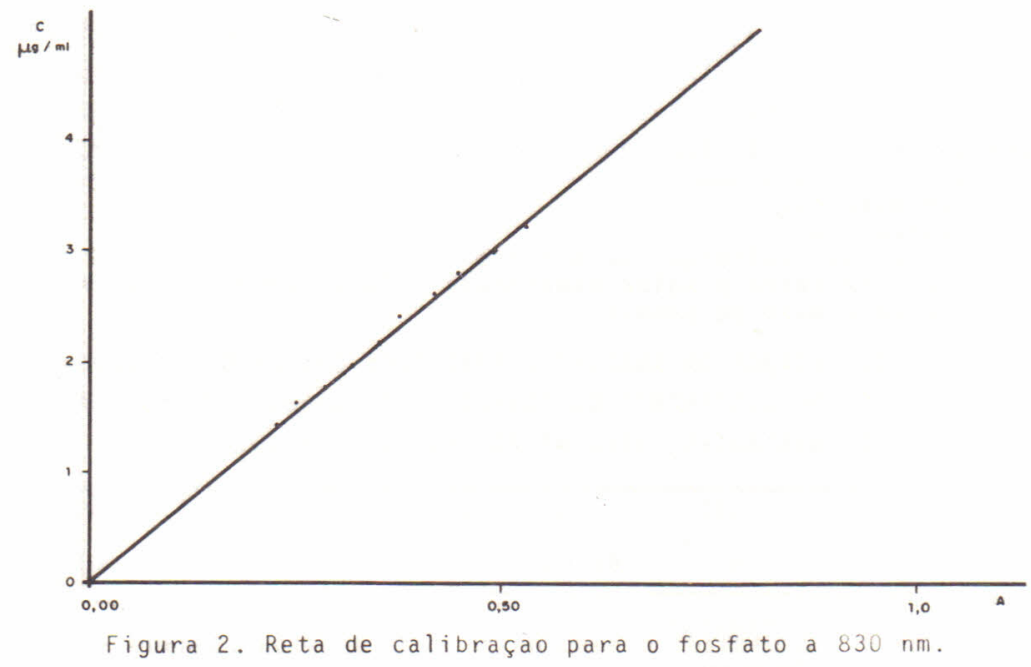

CONCLUSOES

Nas condições experimentais do presente trabalho, conclui-se que:

no mētodo do azul de molibdēnio para doseamento de fósfó ro, o espectro de absorção apresenta um pico de máxima bem definido em $830 \mathrm{~nm}$;

- intervalo de concentração, no qual o método é válido, es tá entre 1,4 a 3,2 $\mu \mathrm{g}$ de fósforo;

não houve interferéncia das concentrações de ions cloreto, lactato e citrato presentes no medicamento analisado;

- método pode ser aplicado para a anālise de fósforoempre parações reidratantes, conforme ficou demonstrado ao longo deste tra batho. 


\section{BIBLIOGRAFIA CITADA*}

1. AMADOR,E. \& URBAN,J., Clin. Chem., 18, 601 (1972).

2. BARTLETT,G.R., J. Biol. Chem., 234, 466 (1959).

3. CROUCH,S.R. \& MALMSTADT,H.V., Anal. Chem., 39, 1085 (1967).

4. DALY,J.A. \& ERTINGSHAUSEN,G., Clin. Chem., 18, 263 (1972).

5. DICKMAN,S.R. \& BRAY,R.H., Ind. Eng. Chem., 12,665 (1940).

6. DONALD,R., SCHWEHR,E.W. \& WILSON,H.N., J.Sci.Food, 7, 677 (1956).

7. DREWES,P.A., Clin. Chem Acta, 39, 81 (1972).

8. GARRAT,D.C. The Quantitative Analysis of Drugs, Chapman \& Hall, 3rd. ed., London, 1964, p.528.

9. KIRK PATRICK,D.S. \& BISHOP,S.H., Anal. Chem., 43, 1707 (1971).

10. MORIN,L.G. \& PROX,J., Clin. Chem. Acta, 46, 113 (1973).

11. MORRIS,H.J. \& CALVARY,H.0., Ind. Eng. Chem., 9, 447 (1937).

12. OHLWEILLER,0.C. Teoria e Prätica de Análise Quantitativa Inorgâa nica, Ed. Brasilia, Brasilia, 1968, p. 1006.

13. SCHAFFER,F.L., FONG,J. \& KIRK,P.L., Anal. Chem., 25, 343 (1953).

14. SEE,Y.P. \& FITT,P.S. Anal. Biochem., 49, 430 (1972).

15. SHAW,M.C., Anai. Biochem., 44, 288 (1971).

16. WEetMAN,D.A., KENNEDY,J.F., Anal. Chem. Acta, 55, 448 (1971).

Recebido em agosto, 1980; aceito em outubro, 1980. 
